\title{
Comparison of efficacy of HCAG and FLAG re-induction chemotherapy in acute myeloid leukemia patients of low- and intermediate-risk groups
}

\author{
J. Y. Zhang ${ }^{1} \cdot$ K. Yu ${ }^{2}$ D L. J. Li ${ }^{1}$
}

Received: 23 January 2019 / Accepted: 11 March 2019 / Published online: 26 March 2019

(c) The Author(s) 2019

\begin{abstract}
Purpose The aim of the present study was to investigate the efficacy and adverse effects of HCAG and FLAG re-induction chemotherapy in acute myeloid leukemia (AML) patients of low- and intermediate-risk groups following induction failure. Methods A total of 98 AML patients were enrolled. Among these subjects, 47 patients were treated with HCAG chemotherapy, while 51 patients were treated with FLAG chemotherapy.

Result The complete remission (CR) and overall remission (OFF) were $24 \%$ and $38 \%$, respectively in patients with HCAG induction chemotherapy, while the corresponding percentages were $28 \%$ and $42 \%$ in subject receiving FLAG chemotherapy. The median survival time of progress-free survival (PFS) was 29.8 (95\% CI 23.749-35.851) months in the HCAG group and 30.8 (95\% CI 21.728-39.872) months in the FLAG group $(P=0.620)$. A total of 42 patients in the HCAG group suffered from grade 4 hematological toxicity, while this adverse reaction was noted for all patients who were treated with FLAG chemotherapy $(P=0.023)$. A total of 19 cases indicated apparent nonhematological toxicity in the HCAG group, while only $40(78.4 \%)$ were noted with these adverse reactions in the FLAG group $(P=0.000)$.

Conclusion The HCAG regimen exhibited a similar effect compared with the FLAG regimen in low- and intermediate-risk groups, although the HCAG regimen significantly decreased the toxicity compared with that noted in the FLAG regimen group.
\end{abstract}

Keywords HCAG · FLAG · Induction failure · Re-induction chemotherapy · Complete remission rate · Overall survival · Acute myeloid leukemia

\section{Introduction}

Acute myeloid leukemia (AML) is the most common form of acute leukemia. The disease is caused by infiltration of tumor cells in the blood, bone marrow, and other tissues $[1,2]$. Although a variety of chemotherapeutic strategies have been developed, and the induction therapy response

\footnotetext{
K. Yu

172871414@qq.com

J. Y. Zhang

zhangjunyu815@163.com

L. J. Li

lilinjie0394@163.com

1 Lishui Municipal Central Hospital, Lishui, Zhejiang, China

2 Wenzhou Medical University First Affiliated Hospital, Wenzhou, Zhejiang, China
}

is successfully applied, a significant percentage of the patients (approximately $20-40 \%$ of the patients) fail to respond to therapy. The overall survival of the patients with induction therapy failure is significantly low compared with the percentage of patients with induction therapy who are in complete remission. Therefore, more effective therapies are required for the treatment of these patients. According to the National Comprehensive Cancer Network (NCCN) guidelines, the treatment strategy of the patients with induction failure may include targeted agents, combination chemotherapy and immunotherapy. Standard or high dose of cytarabine with other cytotoxic drugs such as fludarabine and granulocyte colony-stimulating factor, can be used. It has been reported that cytarabine plus fludarabine and granulocyte colony-stimulating factor (FLAG) form a salvage therapy in AML patients and can result in complete remission for a percentage range of $46-63 \%$ of the patients [3, 4]. Despite its high CR rate, 
FLAG can cause severe toxicity, such as hematological toxicity, mucositis, renal insufficiency, hepatic dysfunction and infections. The treatment-related mortality (TRM) of such cases was reported to range from 7 to $11.4 \%$ and the major cause of mortality was fungal infections [5, 6]. Therefore, effective and safe regimens are required to improve the survival of these patients. In previous studies, Wang $\mathrm{T}$ et al. compared the $\mathrm{CR}$ rate and toxicity between cytarabine, aclarubicin and granulocyte colonystimulating factor (CAG) and FLAG groups. They showed that the CR rate and oral cavity toxicity were significantly different between these two groups. The CR rate and oral cavity toxicity of the FLAG group were higher than those of the CAG's group [6]. Homoharringtonine (HHT) is one of the anti-tumor alkaloids isolated from the Chinese genus Cephalotaxus, and it is a periodic non-specific antitumor drug. HHT was found suitable for various types of AML treatment. The regimen containing HHT is currently widely used for the treatment of AML [7, 8]. Homoharringtonine, aclarubicin and cytarabine (HAA) regimen was reported to achieve $93.3 \% \mathrm{CR}$ rate for AML $(8 ; 21)$ as the first course of induction therapy [7]. In the present study, we used CAG add HHT as one new regimen and compared its efficacy and toxicity with those of the FLAG regimen.

\section{Materials and methods}

The present prospective study was conducted at the Lishui Municipal Central Hospital, which is a University-affiliated hospital with 1680 beds. A total of 98 AML patients with induction failure in low- and intermediate-risk groups were enrolled from May 2011 to July 2018. The research protocol was approved by the Medical Research and Ethics Committee of the hospital. Each patient signed the relevant informed comment. The inclusion criteria were as follows: (1) diagnosis of AML according to the classification of morphological, immunological, cytogenetic and molecular characteristics of white blood cells; (2) identification of risk status for low- and intermediate-risk groups according to the NCCN Guideline Version 2 issued in 2018; (3) initial treatment by standard dose of cytarabine and anthracyclines (standard $3+7$ chemotherapy); (4) the presence of more than $20 \%$ of the treated tumor cells in the bone marrow of the patients during the 14-21 days follow-up period; (5) eastern Cooperative Oncology Group (ECOG) performance score less than 3 ; (6) normal cardiac, liver and renal functions. The exclusion criteria were the following: (1) acute promyelocytic leukemia, (2) serious organ dysfunction, (3) lactation or pregnancy. The patients of our present study were not allowed to attend other clinical trials. The endpoint was the number of patients with relapse.

\section{Treatment}

The present study was a single blind, randomized controlled trial. According to the method of random allocation, all patients treated with HCAG or FLAG re-induction chemotherapy were randomized. The HCAG regimen consisted of the following drugs: low-dose cytarabine $\left(10 \mathrm{mg} / \mathrm{m}^{2}\right)$, at a $12-\mathrm{h}$ dose cycle for days $1-14$, aclarubicin $\left(7 \mathrm{mg} / \mathrm{m}^{2} / \mathrm{d}\right)$ on days $1-8$, G-CSF $\left(200 \mu \mathrm{g} / \mathrm{m}^{2} / \mathrm{d}\right)$ on days $1-14$ and HHT $\left(1.5 \mathrm{mg} / \mathrm{m}^{2} /\right.$ day $)$ on days $1-8$. The FLAG regimen was as follows: fludarabine $\left(30 \mathrm{mg} / \mathrm{m}^{2} /\right.$ day) on days $1-5$, cytarabine ( $2 \mathrm{~g} / \mathrm{m}^{2} /$ day) on days $1-5$, and G-CSF ( $300 \mu \mathrm{g} /$ day) on days $0-5$. The patients who achieved CR continued to receive consolidation therapy according to the NCCN guidelines. The patients with partial remission (PR) or no remission (NR) were administered second or high salvage therapy.

\section{Data collection}

The basement data were the following: age, gender, the percentage of BM blast cells following induction chemotherapy for 14-21 days, disease status, primary or secondary liver function, temperature measurement, chest computed tomography scan, glutamic-pyruvic transaminase (GPT) and glutamic-oxalacetic transaminase (GOT) levels, renal function, creatinine and urea nitrogen levels, and the symptoms nausea, vomiting, and diarrhea.

\section{Definitions and outcomes}

AML risk stratification was assessed according to the 2018 NCCN Guidelines, based on validated cytogenetics and molecular abnormalities. The classification criteria of acute myeloid leukemia were as follows: (1) low risk: the cytogenetics including a core binding factor: inv(16), $\mathrm{t}(16 ; 16)$, $\mathrm{t}(8 ; 21)$. Normal cytogenetics combine with NPM1 mutation positive and FLT3-ITD negative or presence of FLT3ITD with low allelic ratio $(<0.5)$, isolated biallelic CEBPA mutation. (2) Intermediate-risk: normal cytogenetics, $t(9 ; 11)$ and the presence of other non-defined cytogenetics with the exception of poor-risk cytogenetics, or molecular abnormalities including mutated NPM1 and FLT3-ITD with high allelic ratio $(\geq 0.5)$, wild-type NPM1 without FLT3-ITD or with FLT3-ITD low allelic ratio, core binding factor with KIT mutation. A total of 243 cases experienced first induction failure from May 2011 to July 2018. According to this risk stratification, we retrospectively analyzed the cases before 2018, and concluded that a total of 98 cases were eligible for our study. CR status was evaluated in this aspects 
as follows: (1) no evidence indicates abnormal molecular profile and abnormal cytogenetics. (2) BM blast less than 5\% following chemotherapy. (3) peripheral blood (PB): platelet count higher than $100 \times 10^{9} / \mathrm{L}$ or leukocyte count higher than $1 \times 10^{9} / \mathrm{L}$ without transfusion requirements. PR was evaluated as achieved in one of the following conditions: (1) BM blasts 5-25\%. (2) A 50\% or better decrease in BM blasts. (3) BM blasts $<5 \%$ but with Auer rods' presence. The overall remission rate (ORR) was assessed in patients with CR and PR. The induction failure was defined as the failure to achieve ORR following standard $3+7$ regimen treatment on days 14-21. The toxicity was evaluated according to the guidelines provided by the World Health Organization. The toxicity was divided to hematological and nonhematological toxicity, which comprised oral cavity toxicity, gastrointestinal complications, hepatic dysfunction, renal function impairment and pulmonary infection [9].

\section{Observation time}

The median observation time was 30.2 months (range of 21.728-39.872 months).

\section{Statistics}

The data were analyzed using SPSS17. The comparison between HCAG and FLAG was analyzed by the $t$ test. The univariate analysis of $\mathrm{CR}$ was analyzed using the $\chi^{2}$ test. The factors that exhibited significant differences $(P<0.01)$ from the initial analysis were analyzed by multivariate logistic regression. The PFS was estimated by the Kaplan-Meier method and the differences between the two groups were evaluated using the log-rank test. Cox proportional hazard models were constructed for PFS analysis. A stepwise selection method was used to determine the potential confounding covariates. The hazard ratio (HR) was estimated to assess the association of risk factors with PFS. A $P$ value lower than $0.05(P<0.05)$ was considered for significant differences.

\section{Results}

\section{Patient baseline characteristics}

A total of 47 patients were enrolled in the HCAG group. This population contained 25 male and 22 female subjects. The median age was $48 \pm 21.6$ years. In contrast to the HCAG group, 51 patients were enrolled in the FLAG group, which included 32 male and 19 female subjects. The median age was $45 \pm 20.7$ years. The ECOG performance was used for patient evaluation, according to the following score: 0,1 , and 2. The HCAG group indicated that 18 (38.30\%) cases exhibited abnormal chromosome number, while the number of the corresponding cases was $22(43.14 \%)$ in the FLAG group. The BM blast at baseline in the HCAG and FLAG groups was estimated to $45 \pm 22.9$ and $45 \pm 22.9$, respectively. A total of 44 and $50(93.6 \%, 98 \%)$ subjects were de novo AML cases in the HCAG and the FLAG groups, respectively. The remaining three and one $(6.4 \%$ and $4.1 \%)$

\begin{tabular}{llll}
\hline Parameters & $\begin{array}{l}\text { HCAG treatment } \\
(N=47)\end{array}$ & $\begin{array}{l}\text { FALG treatment } \\
(N=51)\end{array}$ & $P$ value \\
\hline Age $($ years $)$ & $48 \pm 21.6$ & $45 \pm 20.7$ & 0.071 \\
Gender $($ male/female $)$ & $25 / 22$ & $32 / 19$ & 0.247 \\
De novo or secondary & & & 0.552 \\
De novo $(n / \%)$ & $44(93.6 \%)$ & $50(98 \%)$ & \\
Secondary $(n / \%)(n / \%)$ & $3(6.4 \%)$ & $1(4.1 \%)$ & \\
Abnormal chromosome $(n / \%)$ & $18(38.30 \%)$ & $22(43.14 \%)$ & 0.749 \\
WBC $\geq 50 \times 10^{9} / \mathrm{L}(n / \%)$ & $13(27.7 \%)$ & $11(21.6 \%)$ & 0.484 \\
HGB $\leq 60$ g/L $(n / \%)$ & $9(19.1 \%)$ & $12(23.5 \%)$ & 0.598 \\
PLT $\leq 30 \times 10^{9} / \mathrm{L}(n / \%)$ & $24(51.1 \%)$ & $30(58.8 \%)$ & 0.146 \\
CEBPA $(n / \%)$ & $1(2.1 \%)$ & $3(5.9 \%)$ & 0.669 \\
c-kit $(n / \%)$ & $3(6.4 \%)$ & $3(5.9 \%)$ & 1.000 \\
NPM1 $(n / \%)$ & $2(4.3 \%)$ & $9(17.6 \%)$ & 0.075 \\
NPM1andFLT3-ITD $(n / \%)$ & $4(8.5 \%)$ & $6(11.8 \%)$ & 0.843 \\
BM blasts $(\%)$ & $45 \pm 22.9$ & $48 \pm 23.2$ & 0.066 \\
CR $(n / \%)$ & $24(51.1 \%)$ & $28(54.9 \%)$ & 0.704 \\
ORR $(n / \%)$ & $38(80.9 \%)$ & $42(82.4 \%)$ & 0.848 \\
Treatment-related mortality(TRM) $(n / \%)$ & $0(0)$ & $4(7.8 \%)$ & 0.119 \\
\hline
\end{tabular}




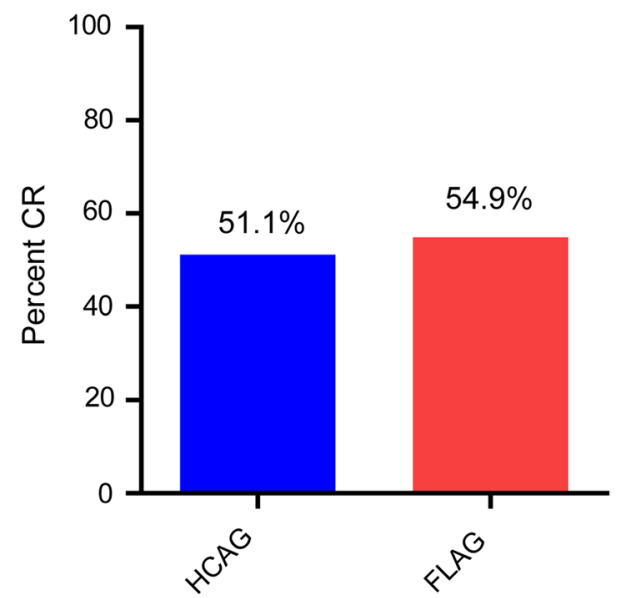

Fig. 1 Clinical efficacy after CLAG and FLAG therapies. 24/47 $(51.1 \%)$ patients in HCAG group were evaluable for remission of which $51.1 \%$ achieved CR; 28/52 (54.9\%) patients in FLAG group were evaluable for remission of which $54.9 \%$ achieved CR. No difference of CR between CLAG and FLAG was observed $(P=0.704)$

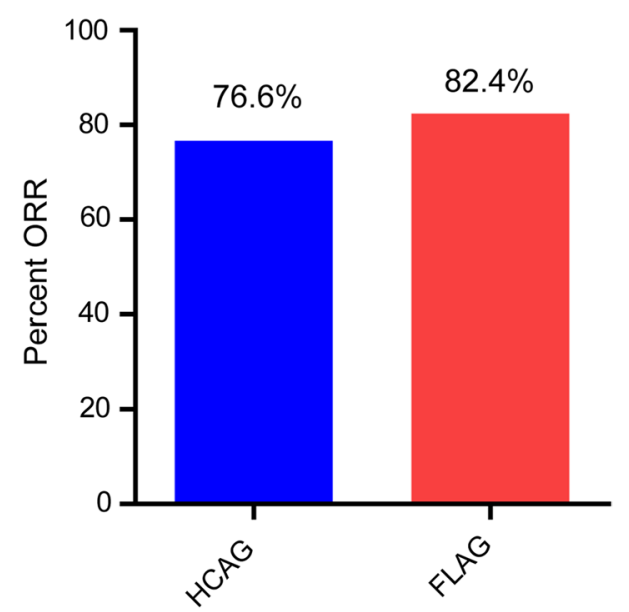

Fig. 2 Clinical efficacy after CLAG and FLAG therapies. 38/47 (80.9\%) patients in HCAG group were evaluable for remission of which $51.1 \%$ achieved ORR; $42 / 52(82.4 \%)$ patients in the FLAG group were evaluable for remission of which $54.9 \%$ achieved ORR. No difference in CR was noted between CLAG and FLAG $(P=0.848)$

subjects were secondary cases in the HCAG and FLAG groups, respectively (Table 1).

\section{Clinical efficacy of the two treatments}

$51.1 \%(24 / 47)$ and $54.9 \%(28 / 51)$ of the patients exhibited CR in the HCAG and FLAG groups, respectively $(P=0.704)$ (Fig. 1). $80.9 \%(38 / 51)$ of the subjects achieved ORR in the HCAG group, while $82.4 \%(42 / 51)$ of these cases were noted in the FLAG group $(P=0.848)$ (Fig. 2). No significant differences were noted between
Table 2 Univariate analysis of CR

\begin{tabular}{ll}
\hline Parameters & $P$ value \\
\hline Age & 0.097 \\
Male (vs. Female) & 0.350 \\
Abnormal chromosome (vs. normal chromosome) & 0.914 \\
Secondary disease (vs.de novo) & 1.000 \\
WBC & 0.353 \\
PLT & 0.029 \\
HGB & 0.101 \\
CEBPA gene positive & 0.120 \\
c-kit gene positive & 1.000 \\
NPM1 gene positive & 0.408 \\
Both NPM1 gene and FLT3-ITD gene positive & 0.643 \\
Hematological toxicity (grade 4) & 0.888 \\
Nonhematological toxicity & 0.009 \\
Oral cavity toxicity (> grade 2) & 0.546 \\
Pulmonary infection & 0.000 \\
Gastrointestinal complications & 0.951 \\
Hepatic dysfunction & 0.227 \\
Renal function impairment & 0.371 \\
BM blasts & 0.002 \\
\hline
\end{tabular}

Table 3 Multivariate logistic regression analysis of CR

\begin{tabular}{lllll}
\hline Parameters & \multicolumn{4}{l}{ Univariate logistic regression } \\
\cline { 2 - 5 } & $P$ value & OR & $95 \%$ CI & \\
\cline { 3 - 5 } & & & Lower & Upper \\
\hline Age & 0.006 & 0.944 & 0.906 & 0.983 \\
Toxicity & 0.603 & 1.523 & 0.313 & 7.412 \\
PLT & 0.947 & 1.000 & 0.994 & 1.006 \\
Pulmonary infection & 0.026 & 0.164 & 0.034 & 0.804 \\
BM blasts & 0.045 & 0.963 & 0.928 & 0.999 \\
\hline
\end{tabular}

the two groups. A total of four patients died for treatment relative adverse reaction and all of them came from FLAG group. The factors affecting CR were evaluated by the $\chi^{2}$ test. (Table 2). The parameters, nonhematological toxicity $(P=0.009)$, pulmonary infection $(P=0.000)$, platelet counts $(P=0.029)$ and BM blasts $(P=0.002)$ were associated with low possibility of achieving $\mathrm{CR}$. The factors age, toxicity, platelet counts, BM blasts, pulmonary infection $(P<0.1)$ were analyzed in the multivariate model. The results indicated that the parameters age $(P=0.006)$, BM blasts $(P=0.014)$, pulmonary infection $(P=0.026)$ were independent predictive factors of CR (Table 3 ). The variables nonhematological toxicity $(P=0.022)$, abnormal chromosome number $(P=0.034)$, pulmonary infection $(P=0.001)$ and BM blasts $(P=0.014)$ were associated with ORR (Table 4). The factors age, nonhematological 
Table 4 Univariate analysis of ORR

\begin{tabular}{ll}
\hline Parameters & $P$ value \\
\hline Age & 0.098 \\
Male (vs. female) & 0.219 \\
Hematological toxicity (grade 4) & 0.585 \\
Abnormal chromosome (vs. normal chromosome) & 0.034 \\
Secondary disease (vs.de novo) & 1.000 \\
WBC & 0.182 \\
PLT & 0.148 \\
HGB & 0.600 \\
CEBPA gene positive & 0.579 \\
c-kit gene positive & 1.000 \\
NPM1 gene positive & 0.874 \\
Both NPM1 gene and FLT3-ITD gene positive & 0.704 \\
Nonhematological toxicity & 0.022 \\
Oral cavity toxicity (> grade 2) & 0.654 \\
Gastrointestinal complications & 0.910 \\
Hepatic dysfunction & 1.000 \\
Renal function impairment & 0.149 \\
Pulmonary infection & 0.001 \\
BM blasts & 0.014 \\
\hline
\end{tabular}

Table 5 Multivariate logistic regression analysis of ORR

\begin{tabular}{llllr}
\hline Parameters & \multicolumn{4}{l}{ Univariate logistic regression } \\
\cline { 2 - 5 } & $P$ value & OR & $95 \%$ CI & \\
\cline { 3 - 5 } & & & Lower & Upper \\
\hline Age & 0.036 & 0.943 & 0.892 & 0.996 \\
Nonhematological toxicity & 0.896 & 1.180 & 0.100 & 13.942 \\
$\begin{array}{l}\text { Abnormal chromosome (vs. } \\
\quad 0.125\end{array}$ & 2.795 & 0.751 & 10.403 \\
normal chromosome) & & & & \\
Pulmonary infection & 0.151 & 0.187 & 0.019 & 1.838 \\
BM blasts & 0.038 & 0.961 & 0.925 & 0.998 \\
\hline
\end{tabular}

toxicity, abnormal chromosome number, pulmonary infection and $\mathrm{BM}$ blasts $(P<0.1)$ were analyzed by the multivariate model. The variables age $(P=0.036)$ and BM blasts $(P=0.038)$ indicated significant differences in the multivariate logistic regression analysis of ORR (Table 5). HCAG resulted in a median PFS of 29.8 (95\% CI 23.749-35.851) months, while FLAG extended PFS to 30.8 (95\% CI 21.728-39.872). The $\mathrm{K}-\mathrm{M}$ curve and the log-rank tests were conducted to analyze PFS (Fig. 3). The data revealed no significant difference in PFS between the HCAG and the FLAG groups $(P=0.620)$. The univariate Cox regression analysis revealed that age $(P=0.000)$, nonhematological toxicity $(P=0.002)$, renal function impairment $(P=0.000)$, pulmonary infection $(P=0.002)$, hematological toxicity grade $4(P=0.004), \mathrm{CR}(P=0.000)$ and ORR $(P=0.000)$ were associated with PFS. The parameter age was associated with shorter PFS and CR, whereas the ORR group exhibited longer PFS (Table 6).

\section{Toxicity}

A total of 42 patients in the HCAG group experienced grade 4 hematological toxicity compared with all the patients in the FLAG group that did not exhibit this symptom $(P=0.023)$. A total of 19 cases indicated considerable nonhematological toxicity in the HCAG group, while 40 patients (78.4\%) exhibited this feature in the FLAG group $(P=0.000)$. No significant differences were noted between the two groups with regard to the hematological or nonhematological toxicities. The toxicity of the HCAG group was lower than that of the FLAG group. The groups were divided into five subgroups based on the difference in nonhematological toxicity. The groups were as follows: oral cavity toxicity, hepatic dysfunction, renal impairment, pulmonary infection and gastrointestinal disorders. A total of eight (17.02\%) cases exhibited oral cavity toxicity higher than grade 2 in the HCAG group, whereas 27 (52.94\%) cases exhibited this type of toxicity in the FLAG group $(P=0.000)$. A total of ten patients demonstrated hepatic dysfunction and were divided to two (4.3\%) cases in the HCAG group and eight (15.7\%) cases in the FLAG group $(P=0.117)$. Renal impairment occurred in four $(8.5 \%)$ patients of the HCAG group and $5(9.8 \%)$ patients of the FLAG group $(P=1.000)$. A total of 47 subjects exhibited pulmonary infection following chemotherapy, of which $15(31.9 \%)$ cases were included in the HCAG group, and $32(62.7 \%)$ in the FLAG group $(P=0.002)$. A total of 13 patients experienced gastrointestinal disorders and were divided to $6(12.8 \%)$ subjects in the HCAG group and 7 $(13.7 \%)$ in the FLAG group $(P=0.889)$ (Table 7).

\section{Discussion}

According to the NCCN guideline, FLAG therapy is considered a salvage therapy for patients of low and intermediate risk for AML following initial induction failure. Various studies have indicated that FLAG can achieve approximately $50 \% \mathrm{CR}$ rate in treating patients with refractory or relapsed AML. In addition, the treatment-related mortality is estimated to $11.4 \%$. Therefore, the toxicity caused by FLAG chemotherapy is substantial. Due to these disadvantages, several alternative regimens of FLAG chemotherapy were used in clinical studies.

Homoharringtonine (HHT) is an alkaloid derived from trees of the genus Cephalotaxus and has demonstrated significant efficacy for the treatment of acute myeloid leukemia (AML). This compound has been widely used in China. The mechanism of action of HHT involves incorporation of the compound into the DNA that causes inhibition of 
Fig. 3 Extended PFS by HCAG and FLAG treatments. K-M curve analysis of accumulating PFS. HCAG therapy achieved median PFS of 29.8 (95\% CI 23.749-35.851) months, whereas FLAG therapy achieved median OS of 30.8 (95\% CI 21.728-39.872) months. No differences were noted with regard to the PFS. The comparison of the PFS between the groups was determined by $\mathrm{K}-\mathrm{M}$ curves and the $\log$-rank test. A $P$ value less than $0.05(P<0.05)$ was considered for significant differences
Table 6 Univariate and multivariate Cox's proportional hazard regression analysis of factors affecting PFS

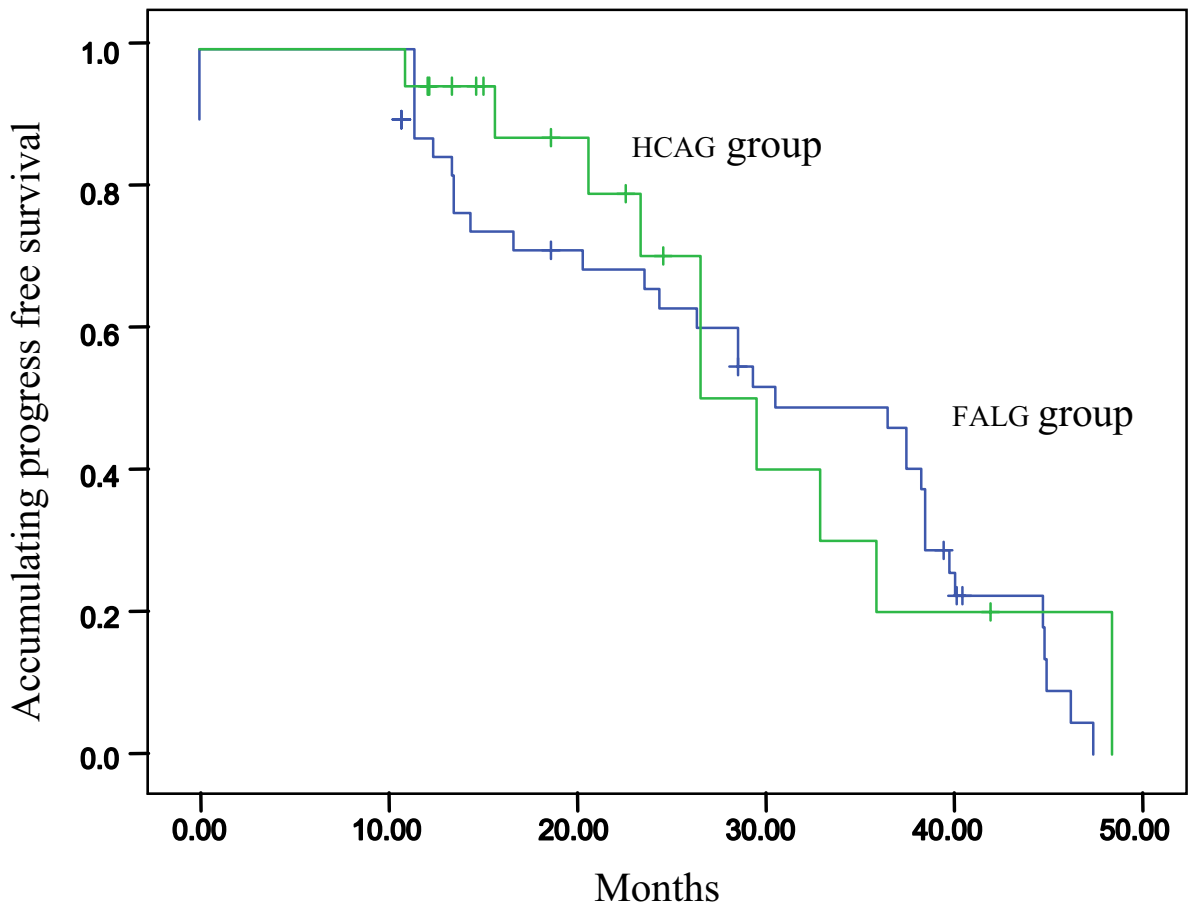

\begin{tabular}{|c|c|c|c|c|c|c|c|c|}
\hline \multirow[t]{3}{*}{ Parameters } & \multicolumn{4}{|c|}{ Univariate Cox regression } & \multicolumn{4}{|c|}{ Multivariate Cox regression } \\
\hline & \multirow[t]{2}{*}{$P$ value } & \multirow[t]{2}{*}{ HR } & \multicolumn{2}{|c|}{$95 \% \mathrm{CI}$} & \multirow[t]{2}{*}{$P$ value } & \multirow[t]{2}{*}{ HR } & \multicolumn{2}{|c|}{$95 \% \mathrm{CI}$} \\
\hline & & & Lower & Higher & & & Lower & Higher \\
\hline Age & 0.000 & 1.094 & 1.059 & 1.130 & 0.000 & 1.152 & 1.099 & 1.207 \\
\hline Male (vs. female) & 0.957 & 1.016 & 0.581 & 1.777 & 0.533 & 0.806 & 0.409 & 1.588 \\
\hline Nonhematological toxicity & 0.002 & 2.564 & 1.400 & 4.697 & 0.783 & 1.254 & 0.251 & 6.252 \\
\hline Oral cavity toxicity (> grade 2 ) & 0.441 & 0.787 & 0.429 & 1.446 & 0.368 & 0.674 & 0.286 & 1.591 \\
\hline Gastrointestinal complications & 0.406 & 1.380 & 0.646 & 2.946 & 0.176 & 2.428 & 0.672 & 8.775 \\
\hline Hepatic dysfunction & 0.143 & 1.902 & 0.805 & 4.492 & 0.061 & 2.771 & 0.953 & 8.061 \\
\hline Renal function impairment & 0.000 & 4.582 & 1.975 & 10.632 & 0.167 & 2.395 & 0.695 & 8.252 \\
\hline Pulmonary infection & 0.002 & 2.453 & 1.389 & 4.333 & 0.119 & 3.363 & 0.731 & 15.464 \\
\hline Hematological toxicity (grade 4) & 0.004 & 0.244 & 0.093 & 0.636 & 0.198 & 0.433 & 0.121 & 1.548 \\
\hline Abnormal chromosome & 0.356 & 0.766 & 0.435 & 1.350 & 0.634 & 1.203 & 0.563 & 2.569 \\
\hline Secondary disease (vs.de novo) & 0.357 & 0.394 & 0.054 & 2.865 & 0.096 & 0.149 & 0.016 & 1.403 \\
\hline WBC & 0.911 & 1.001 & 0.987 & 1.015 & 0.248 & 1.014 & 0.990 & 1.038 \\
\hline HGB & 0.191 & 0.992 & 0.980 & 1.004 & 0.908 & 1.001 & 0.981 & 1.021 \\
\hline PLT & 0.749 & 0.999 & 0.995 & 1.004 & 0.187 & 0.997 & 0.993 & 1.001 \\
\hline CEBPA gene positive & 0.167 & 0.247 & 0.034 & 1.797 & 0.884 & 0.846 & 0.089 & 8.079 \\
\hline c-kit gene positive & 0.634 & 0.753 & 0.234 & 2.420 & 0.820 & 1.173 & 0.298 & 4.618 \\
\hline NPM1 gene positive & 0.596 & 0.805 & 0.362 & 1.791 & 0.296 & 2.588 & 0.436 & 15.377 \\
\hline $\begin{array}{l}\text { Both NPM1 gene and FLT3-ITD } \\
\text { gene positive }\end{array}$ & 0.883 & 0.926 & 0.332 & 2.581 & 0.426 & 0.422 & 0.050 & 3.535 \\
\hline BM blasts & 0.424 & 1.007 & 0.990 & 1.023 & 0.111 & 1.019 & 0.996 & 1.043 \\
\hline CR & 0.000 & 0.152 & 0.081 & 0.284 & 0.001 & 0.235 & 0.098 & 0.562 \\
\hline ORR & 0.000 & 0.153 & 0.080 & 0.291 & 0.001 & 0.177 & 0.061 & 0.512 \\
\hline
\end{tabular}


Table 7 Comparison of two groups with toxicity

\begin{tabular}{llll}
\hline Toxicity & $\begin{array}{l}\text { HCAG treatment } \\
(N=47)\end{array}$ & $\begin{array}{l}\text { FALG treatment } \\
(N=51)\end{array}$ & $P$ value \\
\hline Hematological toxicity (grade 4) & 42 & 51 & 0.023 \\
Nonhematological toxicity & $19(40.4 \%)$ & $40(78.4 \%)$ & 0.000 \\
Oral cavity toxicity $(\geq$ grade 2$)$ & $8(17.02 \%)$ & $27(52.94 \%)$ & 0.000 \\
Gastrointestinal complications $(n / \%)$ & $6(12.8 \%)$ & $7(13.7 \%)$ & 0.889 \\
Hepatic dysfunction $(n / \%)$ & $2(4.3 \%)$ & $8(15.7 \%)$ & 0.117 \\
Renal function impairment $(n / \%)$ & $4(8.5 \%)$ & $5(9.8 \%)$ & 1 \\
Pulmonary infection $(n / \%)$ & $15(31.9 \%)$ & $32(62.7 \%)$ & 0.002 \\
\hline
\end{tabular}

DNA synthesis and induces leukemic cell apoptosis. The decreased expression of the apoptotic-related protein survivin and the down-regulation of the expression of telomerase were also associated with the induction of apoptosis in leukemia cells [10-12]. Moreover, the inhibition of the protein synthesis caused by HHT prevented binding of RNA substrates and aminoacyl-tRNAs to the 60 s ribosomal subunit and inhibited the elongation phase of translation [13]. In addition, HHT can induce leukemic cell differentiation. Therefore, the mechanism of action of HHT is mainly based on the inhibition of protein synthesis, which in turn induces leukemic cell differentiation, inhibits their proliferation, and promotes apoptosis [14-17]. Despite these findings, the exact mechanism remains unclear. HHT can cause synergistic augmentation of therapeutic efficacy towards AML with cytarabine (Ara-c) and aclarubicin (Acla) [15]. Wang et al. demonstrated that HHT had a synergistic relationship with aclarubicin's cytotoxicity in vitro and in vivo [17]. G-CSF is a granulocyle colony-stimulating factor that promotes granulocytic lineage cells from the bone marrow to the peripheral blood and can be used to enhance drug leukemia cell killing. It can also be used to induce G0-G1 phase transition and enhance the anti-leukemic effect of HHT [18]. Previous studies have shown that the CR rate and oral cavity toxicity were significantly different between the FLAG group and CAG group. The CR rate and oral cavity toxicity of the FLAG group were both higher than those of the CAG group [6]. The present study aimed to use a combined treatment of CAG and HHT to produce a new regimen. This regimen was then compared with the FLAG regimen in terms of efficacy and tolerability for AML patients receiving salvage therapy.

The present study compared the efficacy and toxicity of HCAG and FLAG chemotherapy in low- and intermediaterisk groups of AML patients with first induction failure. The results can be summarized as follows: (1) efficacy: no difference was noted in the CR rate between the HCAG and the FALG groups. Specifically, the HCAG patients achieved $51.1 \%$ CR rate, while the FLAG patients $54.9 \%(P=0.704)$ $\mathrm{CR}$ rate. A similar performance was noted with regard to ORR for the two treatments. The patients exhibited $80.9 \%$ ORR in the HCAG and $82.4 \%$ ORR in the FLAG groups, respectively $(P=0.848)$. The PFS exhibited no difference between these two groups and the HCAG group revealed a median PFS of 29.8 (95\% CI 23.749-35.851) months, while the FLAG group a median PFS of 30.8 (95\% CI 21.728-39.872) $(P=0.620)$. Therefore, it can be assumed that no difference was observed in efficacy between the two groups. (2) Toxicity: the HCAG group exhibited lower toxicity than the FLAG group regardless of the grade of hematological toxicity (grade 4$)(P=0.023)$ or the presence of nonhematological toxicity $(P=0.009)$. A total of four cases did not survive in the FALG group while all subjects survived in the HCAG group. Although no significant difference was noted between the two groups, the results suggest that these types of treatment should be handled with caution. In the subgroup of the nonhematological toxicity cases, the parameter incidence rate of oral cavity toxicity (higher than 2), was different between the two groups $(P=0.000)$. In addition, the incidence of pulmonary infection $(P=0.000)$ in the FLAG group was higher than that of the HCAG group. The parameters gastrointestinal complications $(P=0.887)$, hepatic dysfunction $(P=0.778)$ and renal function impairment $(P=1.000)$ were evaluated and no difference was noted between the two groups. This result may be due the low number of cases. Following clinical treatment, non-hematologic toxicity was acceptable and routine supportive therapy was sufficient to control the symptoms.

Based on these results, it can be deduced that the HCAG regimen is as effective as the FLAG regimen and that it is more easily tolerated than the FLAG regimen, which suggests that it may be an optimal selection for low and intermediate-risk AML patients with first induction failure (3). The biochemical factors that affected CR, ORR, and PFS were analyzed as follows: platelet counts $(P=0.029)$ and $\mathrm{BM}$ blasts $(P=0.002)$. These were considered to affect $\mathrm{CR}$ as determined by univariate logistic regression analysis. However, the variables age $(P=0.006), \mathrm{BM}$ blasts $(P=0.014)$, and pulmonary infection $(P=0.026)$ were independent predictive factors for low $\mathrm{CR}$. The probable predictive factors for ORR were the following: nonhematological toxicity $(P=0.022)$, abnormal chromosome number $(P=0.034)$, pulmonary infection $(P=0.001)$, and BM blasts $(P=0.014)$. 
The factors age, nonhematological toxicity, abnormal chromosome number, pulmonary infection, and BM blasts $(P<0.1)$ were analyzed by the multivariate model. The parameters age $(P=0.036)$ and BM blasts $(P=0.038)$ indicated statistically significant differences in the multivariate logistic regression analysis of ORR. This finding is similar to that noted by $\mathrm{Xu}$ et al. who reported that age and BM blast were independent predictive factors for CR, and that BM was an independent predictive factor for low risk of ORR in refractory or relapsed AML patients [19]. Moreover, our study indicated that age was associated with shorter PFS and that CR and ORR were associated with longer PFS. These findings have been verified by several studies.

The present study has several limitations that are summarized as follows: (1) this was a single-center cohort study; (2) the sample size was relatively small. Since there were just 31 patients have molecular abnormalities, it was not clear whether abnormal molecular analyses and stratification based age could affect the clinical efficacy of the two treatments. (3) The first induction regimens including IA, HA, DA, MA, may have influenced the reliability of the results.

In conclusion, HCAG was equally effective to FLAG in re-induction chemotherapy, whereas it exhibited optimal safety and tolerability in low and intermediate-risk AML patients with first induction failure. HCAG regimen can be considered as a new salvage therapy used in low and intermediate-risk AML patients with first induction failure.

\section{Compliance with ethical standards}

Conflict of interest The authors have declared no conflicts of interest.

Ethical approval All procedures performed in the study were in accordance with the ethical standards of the Institutional and National Research Committee and with the 1964 Helsinki Declaration and its later amendments or comparable ethical standards.

Informed consent Informed consent for the treatment of HCAG regimen and FLAG regimen proposed was obtained from all individual participants included in the study.

Open Access This article is distributed under the terms of the Creative Commons Attribution 4.0 International License (http://creativeco mmons.org/licenses/by/4.0/), which permits unrestricted use, distribution, and reproduction in any medium, provided you give appropriate credit to the original author(s) and the source, provide a link to the Creative Commons license, and indicate if changes were made.

\section{References}

1. Dohner H, Weisdorf DJ, Bloomfield CD. Acute myeloid leukemia. N Engl J Med. 2015;373(12):1136-52.

2. O'Donnell MR, Tallman MS, Abboud CN, Altman JK, Appelbaum FR, Arber DA, Attar E, Borate U, Coutre SE, Damon LE, Lancet J,
Maness LJ, Marcucci G, Martin MG, Millenson MM, Moore JO, Ravandi F, Shami PJ, Smith BD, Stone RM, Strickland SA, Wang ES, Gregory KM, Naganuma M, National Comprehensive Cancer Network. Acute myeloid leukemia, version 2.2016. J Natl Compr Cancer Netw. 2016;11(9):1047-55.

3. Martin MG, Augustin KM, Uy GL, Welch JS, Hladnik L, Goyal $\mathrm{S}$, et al. Salvage therapy for acute myeloid leukemia with fludarabine, cytarabine, and idarubicin with or without gemtuzumab ozogamicin and with concurrent or sequential G-CSF. Am J Hematol. 2009;84(11):733-7.

4. Kim H, Lee JH, Joo YD, Bae SH, Lee JH, Kim DY, et al. A prospective, multicenter phase II study of continuous infusion of FLAG for patients older than 60 years with resistant acute myeloid leukemia: a comparison with intensive younger patients' trial. Eur J Haematol. 2016;96(2):188-97.

5. Lee SR, Yang DH, et al. The clinical outcome of FLAG chemotherapy without idarubicin in patients with relapsed or refractory acute myeloid leukemia. Korean Acad Med Sci. 2009;24:498-503.

6. Wang T, Ma L, et al. The comparative study of the effects between modified FLAG and CAG on relapsed or refractory acute myeloid leukemia. Zhonghua Xue Ye Xue Za Zhi. 2014;35(11):966-9.

7. Zhu HH, Jiang $\mathrm{H}$, et al. Homoharringtonine, aclarubicin and cytarabine (HAA) regimen as the first course of induction therapy is highly effective for acute myeloid leukemia with $t(8 ; 21)$. Leukemia Res. 2016;44:40-4.

8. Fan C, Yu W, et al. Efficacy of HAA regimen in the treatment of 64 patients with refractory/relapsed acute myeloid leukemia. Zhonghua Xue Ye Xue Za Zhi. 2016;37(2):100-4.

9. Alberto P, Anglesio E, Bayssas M, et al. World Health Organization handbook for reporting results of cancer treatment. S Geneva, Switzerland: World Health Organization. 1979;48:16-21.

10. Ye XJ, Lin MF. Homoharringtonine induces apoptosis of endothelium and down-regulates VEGF expression of K562 cells. J Zhejiang Univ Sci. 2004;5(2):230-4.

11. Xie WZ, Lin MF, Huang H, Cai Z. Homoharringtonine-induced apoptosis of human leukemia HL-60 cells is associated with downregulation of telomerase. Am J Chin Med. 2006;34(2):233-44.

12. Cai Z, Bao HY, Lin MF. Correlation between survivin mRNA expression and homoharringtonine induced apoptosis of malignant hematopoietic cells. Chin Med J (Engl). 2005;118(7):548-54.

13. Fresno M, Jimenez A, Vazquez D. Inhibition of translation in eukaryotic systems by harringtonine. Eur J Biochem. 1977;72(2):323-30.

14. Gurel G, Blaha G, Moore PB, Steitz TA. U2504 determines the species specificity of the A-site cleft antibiotics: the structures of tiamulin, homoharringtonine, and bruceantin bound to the ribosome. J Mol Biol. 2009;389:146-56.

15. Lü S, Wang J. Homoharringtonine and omacetaxine for myeloid hematological malignancies. J Hematol Oncol. 2014;7:2 PubMed.

16. Zhang T, Shen S, Zhu Z, Lu S, Yin X, Zheng J, et al. Homoharringtonine binds to and increases myosin-9 in myeloid leukemia. Br J Pharmacol. 2016;173:212-21.

17. Wang L, You LS, Ni WM, Ma QL, Tong Y, Mao LP, et al. $\beta$-Catenin and AKT are promising targets for combination therapy in acute myeloid leukemia. Leuk Res. 2013;37:1329-40.

18. Sugiyama T, Kohara H, Noda M, Nagasawa T. Maintenance of the hematopoietic stem cell pool by CXCL12-CXCR4 chemokine signaling in bone marrow stromal cell niches. Immunity. 2006;25(6):977-88.

19. Xu J, Lv TT, et al. Efficacy of common salvage chemotherapy regimens in patients with refractory or relapsed acute myeloid leukemia A retrospective cohort study. Medicine. 2018;97(39):1-8.

Publisher's Note Springer Nature remains neutral with regard to jurisdictional claims in published maps and institutional affiliations. 